NASA Technical Memorandum 83734

\title{
Acoustic Pressures Emanating from a Turbomachine Stage
}

Sridhar M. Ramachandra

Lewis Research Center

Cleveland, Ohio

Prepared for the Ninth Aeroacoustics Meeting cosponsored by the American Institute of Aeronautics and Astronautics and NASA Williamsburg, Virginia, October 15-17, 1984 
ACOUSTIC PRESSURES EMANATING FROM A TURBOMACHINE STAGE

Sridhar M. Ramachandra

National Aeronautics and Space Administration

Lewis Research Center

Cleveland, Ohio 44135

\section{Abstract}

A knowledge of the acoustic energy emission of each blade row of a turbomachine is useful for estimating the overall noise level of the machine and for determining its discrete frequency noise content. Because of the close spacing between the rotor and stator of a compressor stage, the strong aerodynamic interactions between them have to be included in obtaining the resultant flow field. This paper outlines a three-dimensional theory for determining the discrete-frequency noise content of an axial compressor consisting of a rotor and a stator each with a finite number of blades. The lifting surface theory and the linearized equation of an ideal, nonsteady compressible fluid motion are used for thin blades of arbitrary cross section. The combined pressure field at a point of the fluid is constructed by linear addition of the rotor and stator solutions together with an interference factor obtained by matching them for net zero vorticity behind the stage. The rotor solution is obtained as a Fourier-Bessel series; the stator solution is expressed as a Fourier-Laguerre series. The coefficients of the series and the interaction factor are determined as the eigenvector of a set of algebraic equations in matrix form whose elements comprise the sum-integrals of the rotor and stator eigenfunctions. The resultant pressure field of the stage is the sum of the individual perturbation pressures in the presence of the interaction effects, expressions for which are given'herein.

\section{Introduction}

When the rotor is rotating in a uniform and steady incoming free stream, a point behind the rotor experiences periodicity in the pressure and velocity characteristics, which are propagated as acoustic perturbations. The relative motion between the stator blades and the rotor efflux introduces periodicity in the inflow to the stator. Since the rotor efflux consists of both the helical vortex sheets and the viscous wakes of the rotor blades, the aerodynamic forces and moments on the stator blades and the outlet velocities and pressure of the stator are affected by the flow periodicity. Under subsonic flow conditions, periodicity in the stator flow field also affects the rotor. Reflection, transmission of the acoustic perturbation from the blade surface, and interaction with the duct resonance characteristics also have a strong effect on the resulting acoustic field at a point.

Kemp and Sears 1,2 studied the unsteady flow field of two rows of a two-dimensional thin airfoil cascade in incompressible flow al though they did not dwell on the aeroacoustic aspects. The aeroacoustic field of two-dimensional single cascades was studied by several workers (Lane and Friedman ${ }^{3}, \mathrm{Carta}^{4}$, Carta and Fanti. ${ }^{5}$, Hetherington ${ }^{6}$, Parker $7-9$, Tyler. and $\{0$ frin 10,11, Smith12, Whitehead 13 , Morfey14-16, Mani 17,18 , and Mani and Horvay ${ }^{9}$ ). Káji and

Okazaki20-22 studied the aerodynamic interaction between the two rows of infinite airfoil cascades and observed the discrete-frequency circumferential modes and their duct resonance characteristics. Slutsky 23 solved the discrete-frequency noise generation due to rotor forcing excitation and rotorstator interaction by using a Kemp-Sears mechanism with bound vortices on the rotor and the associated three-dimensional velocity potential; he obtained the spin wave characteristics. Namba 24,25 proposed the lifting surface theory for a single blade row in a compressible three-dimensional flow to calculate the unsteady blade forces and their acoustic power generation. He found that the sound power generated was decreased by increasing the radial nonuniformity of a sinusoidal external disturbance in the circumferential direction. Mariano 26 considered the effect of reflection of the sound field from a plane boundary for a uniform distribution of sound sources.

In an earlier paper27, the author outlined a lifting surface theory for a turbomachine stage in a three-dimensional incompressible flow that uses a distribution of sources and vortices to represent the blades of the two rows: the rotor and the stator. Another paper 28 treats the acceleration potential approach to the rotor-stator combination for compressible flow for both the aerodynamic loading and the acoustic characteristics. The concept of a rotor-stator interaction factor is introduced to isolate their mutual interaction effects, and axially attenuated or wavelike solutions are constructed for the general solution by using the Birnbaum series expansion as modulated by the eigenfunctions of the rotor and stator. Thus the composite solution of the rotor and stator is expressed through Fourier-Bessel and Fourier-Laguerre series expansions that simultaneously satisfy the surface boundary conditions on both the blade rows and arbitrarily match to give zero net resultant vorticity in the wake of the stage. The procedure followed in this paper for two blade rows parallels closely that of $\mathrm{Namba}^{25}$ for one blade row. An additional condition is required, however, to quantify the magnitude of the interference between the two blade rows by matching them optimally. In Section 2 the Green's function is written for the linearized differential equations governing the perturbation pressure field of a pulsating unit pressure monopole placed at a point on a blade in a double Fourier-Bessel and Fourier-Laguerre series for the rotor and stator, respectively. From the monopole solution the pulsating unit pressure dipole solution is obtained in Section 3 as the derivative normal to the blade corresponding to the surface pressure distribution. The chordwise pressure distribution on the blade is represented in Section 4 as a Birnbaum series modulated by the pressure eigenfunctions of the rotor and stator corresponding to the camber and thickness effects. The combined rotor-stator field is matched to give a zero net vorticity condition behind the stage (Section 5), and the coefficients of the Birnbaum expansion are determined by satisfying the flow tangency condition on the blade surface (Section 6) followed by a short discussion in Section 7 of the 
combined pressure field. Because of space limitations, only the highlights of this work are presented herein. No attempt has been made here to consider the reflection and absorption of waves or the natural duct resonance characteristics. Numerical results of the present theory will be reported separately.

The author is deeply grateful for the keen interest evinced by $\mathrm{Dr}$. Carl F. Lorenzo during the formulation of the present research program and for his constant encouragement throughout its execution. Thanks are also due to Dr. Anatole Kurkov for his manifold discussions and useful suggestions on the various parts of the research and to $D r$. James A. Pennline for $h$ is useful suggestions on some aspects of the solution of the Laguerre differential equation. The Aerodynamics and Engine Systems Division of the NASA Lewis Research Center provided the necessary atmosphere for the present work for which the National Research Council provided the financial support.

\section{Eigensolutions of Pulsating Pressure Pole on Rotor and Stator}

We shall consider a stage of an axial compressor (Fig. 1) with the rotor followed by a stator located centrally in an infinite annular duct with an outer diameter such that the clearance between the blade tip and the casing is uniform and negligibly small. We shall consider a compressible nonviscous fluid and assume a finite number of rigid blades $Z_{r}$ and $Z_{s}$ in the rotor and stator, respectively. Assuming that the compressor stage is lightly loaded so that the perturbation pressures are small compared with the free-stream static pressure $p_{\infty}$, the linearized differential equations for the perturbation pressures $p_{r}$ and $p_{s}$ of the rotor and stator are given by

$$
\begin{aligned}
& a_{\infty}^{2}\left(\frac{\partial^{2} p_{r}}{\partial r^{2}}+\frac{1}{r} \frac{\partial p_{r}}{\partial r}+\frac{\partial^{2} p_{r}}{r^{2} \partial \theta^{2}}+\frac{\partial^{2} p_{r}}{\partial z^{2}}\right) \\
& =v_{r}^{2} \frac{\partial^{2} p_{r}}{r^{2} \partial \theta^{2}}+w_{r}^{2} \frac{\partial^{2} p_{r}}{\partial z^{2}}+\frac{\partial^{2} p_{r}}{\partial t^{2}} \\
& +2\left(w_{r} \frac{\partial^{2} p_{r}}{\partial z \partial t}+\frac{v_{r}}{r} \frac{\partial^{2} p_{r}}{\partial \theta \partial t}+\frac{v_{r} w_{r}}{r} \frac{\partial^{2} p_{r}}{\partial \theta \partial z}\right) \quad(1 a) \\
& a_{\infty}^{2}\left(\frac{\partial^{2} p_{s}}{\partial r^{2}}+\frac{1}{r} \frac{\partial p_{s}}{\partial r}+\frac{\partial^{2} p_{s}}{r^{2} \partial \theta^{2}}+\frac{\partial^{2} p_{s}}{\partial z^{2}}\right)=v_{s}^{2} \frac{\partial^{2} p_{s}}{r^{2} \partial \theta^{2}} \\
& +w_{s}^{2} \frac{\partial^{2} p_{s}}{\partial z^{2}}+\frac{\partial^{2} p_{s}}{\partial t^{2}}+2\left(w_{s} \frac{\partial^{2} p_{s}}{\partial z \partial t}+\frac{v_{s}}{r} \frac{\partial^{2} p_{s}}{\partial \theta \partial t}+\frac{v_{s} w_{s}}{r} \frac{\partial^{2} p_{s}}{\partial \theta \partial z}\right)
\end{aligned}
$$

in cylindrical polar coordinates where $\left(0, V_{r}, W_{r}\right)$ and $\left(0, V_{S}, W_{S}\right)$ are assumed to be free-stream velocity components along the radial, peripheral, and axial directions for the rotor and stator, respectively, and $a_{\infty}$ is the free-stream speed of sound. Because the stator is situated downstream of the rotor in a region of rapid change, its inflow components $\left(0, V_{S}, W_{S}\right)$ cannot be defined a priori. However, we assume that the stator inlet conditions correspond approximately to that obtained from the velocity diagram (Fig. 2) so that we can write the components of inflow to the rotor and stator as

$$
\left.\begin{array}{l}
\left(0, v_{r}, w_{r}\right)=\left(0, \Omega r, w_{a}\right) \\
\left(0, v_{s}, w_{s}\right)=\left(0, \Omega r-w_{a} \tan \alpha_{2 r}, w_{a}\right)
\end{array}\right\}
$$

where the axial velocity $W_{a}$ is assumed to be constant throughout the stage.

Equations (1) can be nondimensionalized, with all lengths and time expressed in terms of the rotor tip diameter and characteristic time, and written as

$$
\begin{aligned}
\frac{\partial^{2} p_{r}}{\partial r_{1}^{2}}+ & \frac{1}{r_{1}} \frac{\partial p_{r}}{\partial r_{1}}+\left(1-\bar{M}^{2} r_{1}^{2}\right) \frac{\partial^{2} p_{r}}{r_{1}^{2} \partial \theta^{2}}+\beta^{2} \frac{\partial^{2} p_{r}}{\partial z_{1}^{2}} \\
& -M^{2} \frac{\partial^{2} p_{r}}{\partial t_{1}^{2}}-2\left(M^{2} \frac{\partial^{2} p_{r}}{\partial z_{1} \partial t_{1}}+M \bar{M} \frac{\partial^{2} p_{r}}{\partial \theta \partial t_{1}}+M \bar{M} \frac{\partial^{2} p_{r}}{\partial \theta \partial z_{1}}\right) \\
\frac{\partial^{2} p_{s}}{\partial r_{1}^{2}}+ & \left.-\frac{1}{r_{1}} \frac{\frac{\partial p_{s}}{\partial r_{1}}+\left[1-\vec{p}_{r}\right)}{r} e^{i \omega_{r} t_{1}}-\left(M_{2}-M r_{1}\right)\right] \frac{\partial^{2} p_{s}}{r_{1}^{2} \partial \theta^{2}}+\beta^{2} \frac{\partial^{2} p_{s}}{\partial z_{1}^{2}} \\
& -M^{2} \frac{\partial^{2} p_{s}}{\partial t_{1}^{2}}-2\left\{M^{2} \frac{\partial^{2} p_{s}}{\partial z_{1} \partial t_{1}}-\frac{M\left(M_{2}-\bar{M} r_{1}\right)}{r_{1}} \frac{\partial^{2} p_{s}}{\partial \theta \partial t_{1}}\right. \\
& -\frac{M\left(M_{2}-\bar{M} r_{1}\right)}{r_{1}} \frac{\partial^{2} p_{s}}{\partial \theta \theta_{1} \partial z_{1}}=-\frac{\delta\left(\vec{r}_{1}-\vec{p}_{s}\right)}{r_{1}} e^{i \omega_{s} t_{1}}
\end{aligned}
$$

where

$$
\left.\begin{array}{l}
r_{1}=r / r_{t r} ; z_{1}=z / r_{t r} ; t_{1}=t / t_{0} ; \beta^{2}=1-M^{2} ; \\
\beta_{2}^{2}=1-M_{2}^{2} ; t_{0}=r_{t r} / W_{a} ; V_{r}=\Omega r ; M=W_{a} / a_{\infty} ; \\
M_{2}=M \tan \alpha_{2 r} ; M=\Omega r_{t r} / a_{\infty} .
\end{array}\right\}
$$

Equations (3) express the pressure perturbation field of a pulsating unit pressure pole situated on a rotor or stator blade at the vector point $\vec{\rho}_{r}$ or $\vec{\rho}_{s}$ with a pulsation frequency $\omega_{r}$ or $\omega_{s}$, respectively. We may write the general solutions of Eqs. (3) as in $\mathrm{Namba}^{25}$

$$
\begin{array}{r}
\hat{p}_{r}\left(\vec{r}_{1}, \vec{\rho}_{r}\right)=\frac{1}{4 \pi^{2}} \sum_{k_{r}=-\infty}^{\infty} \int_{-\infty}^{\infty} p_{r}\left(r_{1}, o_{r} ; k_{r}, a_{r}\right) \\
\quad x \exp i\left[k_{r}\left(\theta-\varphi_{r}\right)+a_{r}\left(z_{1}-\zeta_{r}\right)\right] d a_{r}
\end{array}
$$




$$
\begin{array}{r}
\hat{p}_{s}\left(\vec{r}_{1}, \vec{\rho}_{s}\right)=\frac{1}{4 \pi^{2}} \sum_{k_{s}=-\infty}^{\infty} \int_{-\infty}^{\infty} p_{s}\left(r_{1}, p_{s} ; k_{s}, a_{s}\right) \\
x \exp i\left[k_{s}\left(\theta-\varphi_{s}\right)+a_{s}\left(z_{1}-s_{s}\right)\right] d a_{s}
\end{array}
$$

where $a_{r}$ and $a_{s}$ are the rotor and stator axial wave numbers; $k_{r}$ and $k_{s}\left(k_{r}, k_{s}=0,+1,+2, \ldots,+\infty\right)$ are the pair of integer separation constants; añd $P_{r}$ and $P_{S}$ are the unknown pressure functions.

We shall express the delta functions in

Eqs. (3) in a combined Fourier series - Fourier integral form as

$$
\begin{aligned}
& \delta\left(\theta-\varphi_{r} ; z_{1}-\zeta_{r}\right) \\
& =\frac{1}{4 \pi^{2}} \sum_{k_{r}=-\infty}^{\infty} \int_{-\infty}^{\infty} \exp i\left[k_{r}\left(\theta-\varphi_{r}\right)+a_{r}\left(z_{1}-\zeta_{r}\right)\right] d a_{r}
\end{aligned}
$$

$$
\begin{aligned}
& \delta\left(\theta-\varphi_{S} ; z_{1}-\zeta_{S}\right) \\
& \quad=\frac{1}{4 \pi^{2}} \sum_{k_{S}=-\infty}^{\infty} \int_{-\infty}^{\infty} \exp i\left[k_{s}\left(\theta-\varphi_{S}\right)+a_{s}\left(z_{1}-\zeta_{S}\right)\right] d a_{S}
\end{aligned}
$$

and write $p_{r}$ and $p_{s}$ as

$$
\left.\begin{array}{l}
p_{r}\left(\vec{r}_{1}, \vec{p}_{r}, t_{1}\right)=\hat{p}_{r}\left(\vec{r}_{1}-\vec{p}_{r}\right) e^{i \omega_{r} t_{1}} \\
p_{s}\left(\vec{r}_{1}, \vec{p}_{s}, t_{1}\right)=\hat{p}_{s}\left(\vec{r}_{1}-\vec{\rho}_{s}\right) e^{i \omega_{s} t_{1}}
\end{array}\right\}
$$

Combining Eqs. (3), (5), (6), and (7) and equating the corresponding terms on the two sides, we obtain the nonhomogeneous second-order ordinary differential equations for the radial pressure functions $P_{r}$ and $P_{S}$, given by

$$
\begin{array}{r}
\frac{d^{2} P_{r}}{d r_{1}^{2}}+\frac{1}{r_{1}} \frac{d P_{r}}{d r_{1}}+\left[\frac{\hat{M}^{2}}{\beta^{2}}-\beta^{2}\left(a_{r}-\frac{M \hat{M}}{\beta^{2}}\right)^{2}-\frac{k_{r}^{2}}{r_{1}^{2}}\right] P_{r} \\
=-\frac{\delta\left(r_{1}-\rho_{r}\right)}{r_{1}}
\end{array}
$$

$$
\begin{aligned}
& \frac{d^{2} \tilde{p} s}{d r_{1}^{2}}+\tilde{p}_{s}\left(-\frac{v\left(k_{s}\right)^{2}}{4}-\frac{x^{(k s)^{2}}}{r_{1}}+\frac{\frac{1}{4}-\bar{\alpha}^{\left(k_{s}\right)^{2}}}{r_{1}^{2}}\right) \\
& =-\frac{\delta\left(r_{1}-\rho_{s}\right)}{r_{1}^{1 / 2}}
\end{aligned}
$$

where we have defined

$$
\begin{aligned}
& P_{s}=\frac{\tilde{P}_{s}}{r_{1}^{1 / 2}} \lambda^{\left(k_{r}\right)^{2}}=\beta^{2}\left[\frac{\hat{M}^{2}}{\beta^{4}}-\left(a_{r}-\frac{M \hat{M}}{\beta^{2}}\right)^{2}\right] \\
& \bar{a}^{\left(k_{s}\right)}=\beta_{2} k_{s} v^{\left(k_{s}\right)^{2}}=4 \beta^{2}\left[\left(a_{s}-M M_{\star}\right)^{2}-M_{\star}^{2}\right] \\
& \hat{M}=\left(M \omega_{r}+M k_{s}\right) \quad x^{\left(k_{s}\right)}=2 M_{2} k_{s}\left(\bar{M} k_{s}+M \omega_{s}+M a_{s}\right) \\
& M_{\star}=\frac{M \omega_{s}+M k_{s}}{\beta^{2}}
\end{aligned}
$$

An examination of Eqs. (3) or (8) shows that when $x \equiv 0$ as in the case of an incompressible fluid or when the exit rotor blade angle $\alpha 2 r=0$ in a compressible fluid, both the equations reduce to the Bessel type. But, for all other cases, $x$ cannot be neglected since $k_{s}$ extends over an infinite range of values, and it is essential to consider $x$ in a formal solution of Eq. (8b). Thus Eq. (8a) is the nonhomogeneous Bessel differential equation and Eq. (8b) is the classical Whittaker nonhomogeneous differential equation, for which the complementary solutions can be written in the form

$$
\begin{aligned}
& P_{r}\left(r_{1}, p_{r}\right)=A_{\star} I\left(\lambda^{\left(k_{r}\right)} r_{1}\right)+B_{\star} K\left(\lambda^{\left(k_{r}\right)} r_{1}\right) \\
& P_{s}\left(r_{1}, \rho_{s}\right)=\left[C_{*} x_{s}\left(k_{s}\right){ }^{\left(v_{s}\right)} r_{1}\right)
\end{aligned}
$$

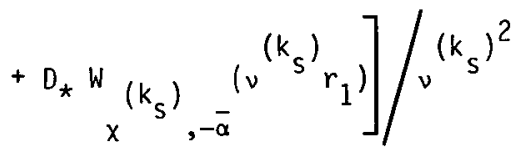

in which $I(x)$ and $K(x)$ represent the modified Bessel and Hankel functions, respectively, and $W_{x, \mu}(x)$ is the Whittaker function. To write the solution of the complete nonhomogeneous equations, it is convenient to express the solution of the rotor function $P_{r}$ in an infinite expansion in terms of the normalized functions $\Phi_{\ell}$ for for Eq. (10a). The function $\Phi_{\ell}^{\left(k_{r}\right)}$ is defined in the appendix. The solution for the stator function $P_{S}$ is more conveniently written in terms of the set of generalized Laguerre functions that satisfy certain orthogonality relations than directly in terms of the Whittaker function, to which they are related by the expressions

$$
\begin{aligned}
W_{x, \mu}(z)=\frac{\Gamma(1+2 \mu) \Gamma\left(x-\mu+\frac{1}{2}\right)}{\Gamma\left(x+\mu+\frac{1}{2}\right)} \\
\quad x e^{z / 2} z(\mu+1 / 2) L_{(x-\mu-1 / 2)}^{(2 \mu)}(z)
\end{aligned}
$$

Thus we shall obtain the solutions of the nonhomogeneous Eqs. (8) for $\rho_{r}$ and $\rho_{S}$ in the form 
$P_{r}^{\left(k_{r}\right)}\left(r_{1}, \rho_{r}\right)=\sum_{\ell=0}^{\infty} g_{\ell}^{\left(k_{r}\right)}{ }_{\left(a_{r}\right) \Phi_{\ell}}^{\left(k_{r}\right)}\left(r_{1}\right) \Phi_{\ell}{ }^{\left(k_{r}\right)}\left(\rho_{r}\right)$

$P_{s}^{\left(k_{s}\right)}\left(r_{1}, p_{s}\right)=\sum_{\ell=0}^{\infty} h_{\ell}^{\left(k_{s}\right)}\left(a_{s}\right) \psi_{\ell}{ }^{\left(k_{s}\right)}\left(r_{1}\right)$

$\times \psi_{\ell}^{\left(k_{s}\right)}\left(\rho_{s}\right)\left(r_{2 s} \rho_{2 s}\right)^{\bar{\alpha}+1 / 2} \exp \left(-\frac{r_{2 s}+\rho_{2 s}}{2}\right)$

where $g_{\ell}^{\left(k_{r}\right)}$ and $h_{\ell}^{\left(k_{s}\right)}$ are coefficients of the expansion to be determined. The eigenfunctions $\Phi_{\ell}\left(k_{r}\right)$ and $\psi_{\ell}\left(k_{s}\right)$ are combinations of Bessel and Laguerre functions, respectively, that satisfy

$\frac{d^{2} \Phi_{\ell}^{\left(k_{r}\right)}}{d r_{1}^{2}}+\frac{1}{r_{1}} \frac{d \Phi_{l}^{\left(k_{r}\right)}}{d r_{1}}+\left(\lambda_{\ell}{ }^{\left(k_{r}\right)^{2}}-\frac{k_{r}^{2}}{r_{1}^{2}}\right) \Phi_{\ell}{ }^{\left(k_{r}\right)}=0$

$\frac{d^{2} \psi_{s}^{\left(k_{s}\right)}}{d r_{2 s}^{2}}+\left(\frac{2 \bar{\alpha}+1}{r_{2}}-1\right) \frac{d \psi_{l}^{\left(k_{s}\right)}}{d r_{2 s}}+\frac{\tilde{l}}{r_{2 s}} \psi_{\ell}\left(k_{s}\right)=0$

in which the independent variables $r_{2 s}$ and $\rho_{2 s}$ are defined by

$$
\left.\begin{array}{l}
r_{2 s}=v_{\ell}^{\left(k_{s}\right)}{ }^{r_{1}} \\
\rho_{2 s}=v_{\ell}\left(k_{s}\right) \\
{ }_{s}
\end{array}\right\}
$$

For the Laguerre equation (Eq. 10a), eigensolutions exist if 31,32

$$
\tilde{\ell}=x_{\ell}^{\left(k_{s}\right)}+\frac{v_{\ell}^{\left(k_{s}\right)}}{h_{\ell}^{\left(k_{s}\right.} T}-\left(\bar{a}+\frac{1}{2}\right)
$$

is an integer, and we shall put

$$
\ell_{1}=\tilde{\imath}+\bar{a}+\frac{1}{2}
$$

$\lambda_{\ell}^{\left(k_{r}\right)}$ and $x_{\ell}^{\left(k_{s}\right)}(\ell=0,1,2, \ldots, \infty)$ may be $r e-$ garded as the radial eigenvalues of the rotor and stator since we can write

$$
\frac{\nu_{l}^{\left(k_{s}\right)}}{4 \beta^{2} M_{*}^{2}}=\frac{1}{M^{2}}\left(\frac{x_{l}}{2 M_{*} M_{2} k_{s}}-1\right)^{2}-1
$$

The stator eigenfunction ${ }_{\psi_{\ell}}^{\left(\mathrm{k}_{s}\right)}$ is defined in the appendix. The functions $\phi_{\ell}\left(k_{r}\right)$ and $\Psi_{\ell}\left(k_{s}\right)$ also satisfy the orthogonality relations within the domain of $r_{1}$, for each blade row such that ${ }^{28}$

$$
\int_{n_{r}}^{1} r_{1} \Phi_{\ell}^{\left(k_{r}\right)}\left(r_{1}\right) \Phi_{m}^{\left(k_{r}\right)}\left(r_{1}\right) d r_{1}=\delta_{\ell m}
$$

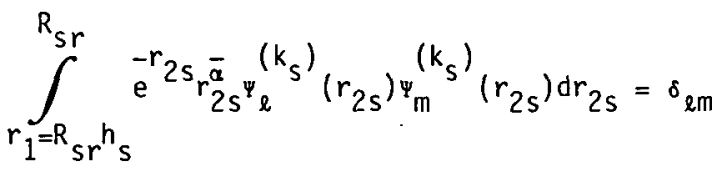

The Dirac delta functions $\delta\left(r_{1}-\rho_{r}\right)$ and $\delta\left(r_{1}-\rho_{s}\right)$ can be expanded in terms of the corresponding eigenfunctions such that

$$
\delta\left(r_{1}-\rho_{r}\right)=\sqrt{r_{1} \rho_{r}} \sum_{\ell=0}^{\infty} \Phi_{\ell}^{\left(k_{r}\right)}{ }_{\left(\rho_{r}\right) \phi_{\ell}}^{\left(k_{r}\right)}\left(r_{1}\right)
$$

$$
\begin{gathered}
\delta\left(r_{1}-\rho_{s}\right)=\sum_{\ell=0}^{\infty} \exp \left(-\frac{r_{2 s}+\rho_{2 s}}{2}\right) \psi_{\ell}{ }^{\left(k_{s}\right)}\left(\rho_{2 s}\right) \\
\times \psi_{\ell}{ }^{\left(k_{s}\right)}\left(r_{2 s}\right)
\end{gathered}
$$

The pressure functions $P_{r}$ and $P_{s}$ must satisfy the end conditions of zero radial flow at both the hub and the tip for which the radial pressure gradients must vanish at both these points and we have the relations

$$
\begin{aligned}
& \frac{d}{d r_{1}} \Phi_{\ell}^{\left(k_{r}\right)}=0 \quad \text { at } r_{1}=h_{r}, r_{1}=1 \\
& \frac{d}{d r_{1}} \psi_{\ell}^{\left(k_{r}\right)}+\left(\frac{\bar{\alpha}}{r_{2 s}}-\frac{1}{2}\right){ }_{\psi}^{\left(k_{s}\right)}=0 \\
& \text { at } r_{2 s}=v_{\ell}^{\left(k_{s}\right)}{ }_{R_{s r}} h_{s} ; r_{2 s}=v_{\ell}^{\left(k_{s}\right)}{ }_{R_{s r}}
\end{aligned}
$$

from which we obtain an infinite set of eigenvalues $\lambda_{\ell}^{\left(k_{r}\right)}, v_{\ell}^{\left(k_{s}\right)}(\ell=0,1,2, \ldots, \infty)$ for each $k_{r}$ and $k_{s}$.

The perturbation pressure amplitudes $\hat{p}_{r}$ and $\hat{p}_{S}$ in Eqs. (5) involve summation over $k_{r}$ and $k_{s}$ the contour so chosen as to yield bounded or exponentially decaying terms in the axial direction. Because of the cumbersome and lengthy nature of the calculations, only the final expressions for subsonic axial flow are given herein as 


$$
\begin{aligned}
& p_{r}\left(\vec{r}_{1}, \vec{\rho}_{r}, t_{1}\right)=\frac{1}{4 \pi \beta} \sum_{\ell=0}^{\infty} \sum_{k_{r}=-\infty}^{\infty} \frac{\ell_{\ell}^{\left(k_{r}\right)}\left(\rho_{r}\right) \Phi_{\ell}^{\left(k_{r}\right)}\left(r_{1}\right)}{\Lambda_{\ell}\left(k_{r}\right)} \\
& x \exp \left\{i\left[k_{r}\left(\theta-\varphi_{r}\right)+\omega_{r} t_{1}+\left(\frac{M \hat{M}}{\beta^{2}}\right) z_{r}\right]-\Lambda_{\ell}{ }^{\left(k_{r}\right)} \frac{\left|\hat{Z}_{r}\right|}{\beta}\right\} \\
& \mathrm{p}_{S}\left(\vec{r}_{1}, \stackrel{\rho}{s}_{S}, t_{j}\right)=\frac{i \beta^{2} M}{\pi} \sum_{\ell=0}^{\infty} \sum_{k_{S}=-\infty}^{\infty} \frac{M_{\star}^{2} \mu}{\left(\beta^{2}-\mu^{2} M^{2}\right)^{\Delta} 1^{\ell} 1} \\
& x \exp \left[-\frac{r_{2 s}+\rho_{2 s}}{2}\right]\left(r_{2 s} \rho_{2 s}\right)^{\bar{\alpha}+\frac{1}{2}} \psi_{\ell}^{\left(k_{s}\right)}{ }_{\left(\rho_{2 s}\right) \psi_{\ell}}^{\left(k_{s}\right)}\left(r_{2 s}\right) \\
& x \exp i\left[k_{S}\left(\theta-\varphi_{S}\right)+\omega_{S} t_{1}\right] \\
& x\left(C F \cos M_{\Delta_{1}} \hat{Z}_{s}+S F \sin \cdot M_{\Delta} \hat{Z}_{s}\right) \\
& x \exp \left[i M M_{\star} \hat{z}_{s}+M\left(i \mathscr{R e} \Delta_{2}-\left|f m \Delta_{2}\right|\right) \mid \hat{Z}_{s}{ }^{\prime}\right]
\end{aligned}
$$

where

$\mu=\frac{M_{2} k}{l_{1}} ; \Delta=1+\frac{\mu}{\left(\beta^{2}-M_{\mu}^{2}\right)} ; \hat{Z}_{S}=\left(z_{1}-\zeta_{S}\right) ;$

$\hat{z}_{r}=\left(z_{1}-\zeta_{s}\right) ; \Delta_{2}=\Delta-1$;

$\Delta_{1}=\left[\left(1-\mu^{2}\right)+\beta^{4} \frac{\left(1+\mu^{2}\right)}{M^{2} \mu^{2}}\right] \times \frac{\mu}{\left(\beta^{2}-M^{2} \mu^{2}\right)}$

\section{Representation of the Lifting Surface}

The lifting surface of the rotor and stator blades $c$ an be regarded as composed of a distribution of pulsating pressure dipoles with constant interpole phase angle factors or and $\sigma_{s}$ and with the dipole axes normal to the local blade surface. The position of the unit dipole on the blade has the coordinates $\left[\rho_{r}, \overline{\varphi_{r}}+\varphi_{r}+m_{r}\left(\sigma_{r}+\delta_{r}\right)\right.$; $\left.z_{r}+\zeta_{r}\right] ;\left[\rho_{s}, \bar{\varphi}_{s}+\varphi_{s}+m_{s}\left(\sigma_{s}+\delta_{s}\right) ; z_{s}+\zeta_{s}\right]$ on the $\left(m_{r}\right)^{\text {th }}$ rotor and $\left(m_{s}\right)$ th stator blade, respectively, in which $\varphi_{r}$ and $\varphi_{S}$ are the corresponding offset angles of the first blade (Fig. 3). The dipole field can be obtained from the pole field by differentiating along the local unit normal to the surface. The field of the $Z_{r}$ and $Z_{s}$ unit pressure dipoles on the blades of the rotor and stator can be obtained by summing over $m_{r}$ and $m_{s}$. If $\Delta p_{r}$ and $\Delta p_{s}$ be the net upward-acting pressure at the points $\vec{\rho}_{r}\left(\rho_{r}, \psi_{r}, \zeta_{r}\right)$ and $\vec{\rho}_{s}\left(\rho_{s}, \psi_{s}, \zeta_{s}\right)$, we can obtain the resulting pressure field of the rotor and stator each in isolation by integrating the dipole field of the intensity $\Delta p_{r}$ or $\Delta p_{s}$ and integrating over the entire surface of each blade with respect to $\rho$ and $\zeta$. For subsonic axial flow, we have the rotor and stator pulsating dipole pressure field given by

$$
\begin{aligned}
& P_{R}\left(\vec{r}_{1}, t_{1}\right) \\
& =-\frac{z_{r}}{4 \pi \beta} \int_{h_{r}}^{1} \int_{r_{1}}^{z_{r}} \sum_{\ell=0}^{\infty} \sum_{k_{r}=-\infty}^{\infty} \Delta p_{r_{\star}}\left(r_{1}, \vec{p}_{r}, t_{1} ; \ell, k_{r}\right) \\
& d \rho_{r} d \zeta_{r} \\
& P_{S}\left(\vec{r}_{1}, t_{1}\right) \\
& =\frac{i \beta^{2} M Z_{s}}{4 \pi} \int_{R_{s r} h_{s}}^{R_{s r}} \int_{z_{s 1}}^{z_{s 2}} \sum_{\ell=1}^{\infty} \sum_{k_{s}=-\infty}^{\infty} \Delta P_{s} \psi_{\star}\left(\vec{r}_{1}, \vec{\rho}_{s}, t_{1} ; \ell, k_{s}\right) \\
& d \rho_{s} d \zeta s
\end{aligned}
$$

where the functions $\Phi_{\star}$ and $\Psi_{\star}$ are given in the appendix.

\section{Effect of Camber and Thickness}

The pressure loading $\Delta \mathrm{p}_{\mathrm{r}}$ and $\Delta \mathrm{p}_{\mathrm{S}}$ on the blades in isolation is caused by the combined effects of blade thickness, camber, and incidence and varies over the blade surface. It can be expressed by combining, it with the, rotor and stator eigenfunctions $\Phi_{\ell}\left(k_{r}\right)$ and $\psi_{\ell}\left(k_{s}\right)$ as

$$
\begin{aligned}
& \Delta p_{r}=\sum_{\ell=0}^{\infty} H_{1}\left(\tilde{\omega}_{r}, \tilde{\rho}_{r} ; k_{r}, \ell\right)=\sum_{\ell=0}^{\infty}\left[F_{1, \ell}\left(k_{r}\right){\tilde{\left(\omega_{r}\right.}}\right) \\
& \left.+F_{2, \ell}^{\left(k_{r}\right)}\left(\tilde{\omega}_{r}\right)\right]_{\ell}^{\left(k_{r}\right)}\left(\rho_{r}\right) \\
& \Delta p_{s}=\sum_{\ell=0}^{\infty} H_{2}\left(\tilde{\omega}_{s}, \rho_{s} ; k_{s}, \ell\right)=\sum_{\ell=0}^{\infty}\left[G_{1, \ell}\left(k_{s}\right){ }_{\left(\tilde{\omega}_{s}\right)}\right. \\
& \left.+G_{2, \ell}^{\left(k_{s}\right)}\left(\tilde{\omega}_{s}\right)\right] \Phi_{\ell}^{\left(k_{s}\right)}\left(\rho_{s}\right)
\end{aligned}
$$

where $\left(F_{1}, F_{2}\right)$ and $\left(G_{1}, G_{2}\right)$ are the chordwise variations for the rotor and stator due to the thickness and camber, respectively, in terms of the chordwise coordinates $\widetilde{\omega}_{r}$ and $\widetilde{\omega}_{s}$ related to the locally orthogonal coordinates $\left(y_{r}^{\prime}, z_{r}\right)$ and $\left(y_{s}^{\prime}, z_{s}\right)$ defined by

$$
y_{r}^{\prime}=-C_{R} \cos \tilde{\omega}_{r} \quad-C_{r} \leq y_{s}^{\prime} \leq+C_{R} \quad 0 \leq \tilde{\omega}_{r} \leq \pi
$$

$$
y_{s}^{\prime}=+C_{S} \cos \tilde{\omega}_{s} \quad-c_{s} \leq y_{s}^{\prime} \leq+C_{S} \quad 0 \leq \tilde{\omega}_{s} \leq \pi
$$

The orthogonal coordinates $\left(r, y_{r}^{\prime}, z_{r}^{\prime}\right)$ and $\left(r, y_{S}^{\prime}, z_{S}^{\prime}\right)$ are related to the Cartesian coordinates $(X, Y, Z)$ by rotation through the angles $\left(\alpha_{r}, \alpha_{s}\right)$ and $\left(\psi_{r}, \psi_{s}\right)$ described in Eq. (26) 
$\left(\begin{array}{c}r \\ y_{r}^{\prime} \\ z_{r}^{\prime}\end{array}\right)=\left(\begin{array}{ccc}\cos \psi_{r} & \sin \psi_{r} & 0 \\ -\sin \psi_{r} \sin \alpha_{r} & \cos \psi_{r} \sin \alpha_{r} & \cos \alpha_{r} \\ \sin \psi_{r} \cos \alpha_{r} & -\cos \psi_{r} \cos \alpha_{r} & \sin \alpha_{r}\end{array}\right)\left(\begin{array}{l}x \\ y \\ z\end{array}\right)$

(26a)

$\left(\begin{array}{c}r \\ y_{s}^{\prime} \\ z_{s}^{\prime}\end{array}\right)=\left(\begin{array}{ccc}\cos \psi_{s} & \sin \psi_{s} & 0 \\ -\sin \psi_{s} \sin \alpha_{s} & \cos \psi_{s} \sin \alpha_{s} & \cos \alpha_{s} \\ \sin \psi_{s} \cos \alpha_{s} & -\cos \psi_{s} \cos \alpha_{s} & \sin \alpha_{s}\end{array}\right)\left(\begin{array}{l}x \\ y \\ z\end{array}\right)$

(26b)

The axial positions of the leading and trailing edges of the blades are given by

$z_{r 1}=z_{r 0}-C_{R} \cos \alpha_{r} ; z_{r 2}=z_{r 0}+C_{R} \cos a_{r}$

$z_{s 1}=z_{s 0}-C_{R} \cos \alpha_{s} ; z_{s 2}=z_{s 0}+C_{S} \cos \alpha_{s}$

where $\left(\psi_{r}, \psi_{s}\right)$ and $\left(\zeta_{r}, \zeta_{s}\right)$ are related to $\omega_{r}$ by the equations

$$
\begin{aligned}
& \psi_{r}=\bar{\varphi}_{r}+\frac{2 \pi m_{r}}{Z_{r}}-\cos ^{-1}\left(\frac{2 C_{R} \sin \alpha_{r} \cos \tilde{\omega}_{r}}{\rho_{r}}\right) \\
& \psi_{s}=\bar{\varphi}_{s}+\frac{2 \pi m_{s}}{Z_{s}}-\cos ^{-1}\left(\frac{2 C_{s} \sin \alpha_{s} \cos \tilde{\omega}_{s}}{\rho_{s}}\right) \\
& \zeta_{r}=Z_{r, 0}+2 c_{R} \cos \alpha_{r} \cos \tilde{\omega}_{r} \\
& { }_{s}=Z_{s, 0}+2 c_{s} \cos \alpha_{s} \cos \tilde{\omega}_{s}
\end{aligned}
$$

The offset angle $\bar{\varphi}_{r}$ of the first blade on the rotor can be written as $\bar{\varphi}_{r}=\Omega_{0} t_{1}$, where $\Omega_{0}=\Omega / t_{0}$; the offset angle $\bar{\varphi}_{s}$ of the first blade on the stator can be set equal to zero. The terms $\left(2 \pi m_{r} / Z_{r}\right)$ and $\left(2 \pi m_{r} / Z_{s}\right)$ represent the polar angles of the $\left(m_{r}\right)^{\text {th }}$ rotor and $\left(m_{s}\right)^{\text {th }}$ stator blades, respectively, at the midpoint of the blade chord. The last terms in $\psi_{r}$ and $\psi_{s}$ give the increment in the polar angles for points $\left(y^{\prime}, z^{\prime}\right)$ away from the midchord point at any radius. We shall represent the functions $\left(F_{1}, F_{2}\right)$ and $\left(G_{1}, G_{2}\right)$ in an infinite trigonometric series expansion corresponding to the Glauert/Birnbaum series as

$$
F_{1, \ell}^{\left(k_{r}\right)}\left(\tilde{\omega}_{r}\right)=A_{0}^{\left(k_{r}\right)} \cot \frac{\tilde{\omega}_{r}}{2}+\sum_{m=1}^{\infty} A_{m}^{\left(k_{r}\right)} \sin \tilde{\omega}_{r}
$$

$$
\begin{aligned}
F_{2, l}^{\left(k_{r}\right)}\left(\tilde{\omega}_{r}\right)=B_{0}^{\left(k_{r}\right)}\left(\cot \frac{\tilde{\omega}_{r}}{2}-2 \sin \tilde{\omega}_{r}\right) \\
+\sum_{m=2}^{\infty} B_{m}^{\left(k_{r}\right)} \sin \tilde{\omega}_{r}
\end{aligned}
$$

$$
\begin{aligned}
G_{1, \ell}^{\left(k_{s}\right)}\left(\tilde{\omega}_{s}\right)= & C_{0}^{\left(k_{s}\right)} \cot \frac{\tilde{\omega}_{s}}{2}+\sum_{m=1}^{\infty} C_{m}^{\left(k_{s}\right)} \sin \tilde{\omega}_{s} \\
G_{2, \ell}\left(k_{s}{ }^{\prime}\left(\tilde{\omega}_{s}\right)=\right. & D_{0}\left(k_{s}\right)\left(\cot \frac{\tilde{\omega}_{s}}{2}-2 \sin \tilde{\omega}_{s}\right) \\
& +\sum_{m=2}^{\infty} D_{m}^{\left(k_{s}\right)} \sin \tilde{\omega}_{s}
\end{aligned}
$$

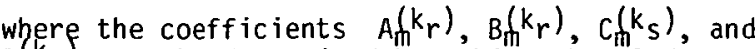
$\left.D_{m} k_{s}\right) \quad c$ an be determined by making the blade surfaces as stream surfaces. The series in Eqs. (29) satisfy the Kutta condition at the blade trailing edge and have the usual singularity at the leading edge. It can be observed from Eqs. (24) that thickness and camber effects are inseparable and are interwoven. Note that the thickness effect corresponds to the monopole solution and can be obtained directly.

\section{Boundary Conditions}

By defining the mean camberline and thickness shapes of the blades through the equations

$$
\left.\begin{array}{l}
z_{C_{r}}^{\prime}=z_{C_{r}}^{\prime}\left(y_{r}^{\prime}\right) ; z_{C_{s}}^{\prime}=z_{C_{s}}^{\prime}\left(y_{s}^{\prime}\right) \\
z_{T_{r}}^{\prime}=z_{T_{r}}^{\prime}\left(y_{r}^{\prime}\right) ; z_{T_{s}}^{\prime}=z_{T_{s}}^{\prime}\left(y_{s}^{\prime}\right)
\end{array}\right\}
$$

The upper and lower surface blade profiles are given by

$$
\left.\begin{array}{l}
z_{U r}^{\prime}=z_{C r}^{\prime}+z_{T r}^{\prime} ; z_{L r}^{\prime}=z_{C r}^{\prime}-z_{T r}^{\prime} \\
z_{U S}^{\prime}=z_{C s}^{\prime}+z_{T s}^{\prime} ; z_{L s}^{\prime}=z_{C s}^{\prime}-z_{T s}^{\prime}
\end{array}\right\}
$$

If $\vec{U}_{r}$ and $\vec{U}_{s}$ be the resultants of the freestream and induced velocity perturbations of the rotor-stator flow field, we can represent the surface flow tangency condition on the upper and lower blade surfaces by the equations

$$
\tau_{U 1}=\left(\frac{d z_{U}^{\prime}}{d y^{\prime}}\right)_{r}=\left(\frac{u_{R z^{\prime}}}{u_{R y^{\prime}}}\right)_{z_{r}^{\prime}=0^{+}} ; \tau_{U 2}=\left(\frac{d z_{U}^{\prime}}{d y_{S}^{\prime}}\right)=\left(\frac{u_{S z^{\prime}}}{u_{S y^{\prime}}}\right)_{z_{S}^{\prime}=0+}
$$

$\tau_{L 1}=\left(\frac{d z_{L}^{\prime}}{d y^{\top}}\right)_{r}=\left(\frac{u_{R z^{\prime}}}{u_{R y^{\prime}}}\right)_{z_{r}^{\prime}=0-} ; \tau_{L 2}=\left(\frac{d z_{L}^{\prime}}{d y^{\top}}\right)_{S}=\left(\frac{u_{S z^{\prime}}}{u_{S y^{\prime}}}\right)_{z_{S}^{\prime}=0-}$

In keeping with the thin airfoil theory postulates, the boundary conditions are satisfied on the blade chord.

To obtain the induced velocity perturbations of the rotor and stator, it is convenient to introduce a local intrinsic helical orthogonal coordinate system (Fig. 4) for each based on the corresponding 
undisturbed streamline with the helix angles $\theta_{\mathrm{hl}}$ and $\theta_{\mathrm{h} 2}$ defined by

$$
\begin{gathered}
\tan \theta_{h 1}=\frac{R_{\star}}{r_{1}} \\
\tan \theta_{h 2}=\frac{R_{\star}}{\left(r_{1}-M_{2} / M\right)}
\end{gathered}
$$

The helical intrinsic coordinates $\left(\rho_{r}, \tau 1, s_{1},\right)$ and $\left(\rho_{s}, r_{2}, s_{2}\right)$ of the rotor and stator are related to the cylindrical coordinates by the matrix transformations of differential lengths as

$$
\begin{aligned}
& \left(\begin{array}{l}
d r \\
d \theta \\
d z
\end{array}\right)=\left(\begin{array}{ccc}
1 & 0 & 0 \\
0 & \sin \theta_{h 1} & -\cos \theta_{h 1} \\
0 & \cos \theta_{h 1} & \sin \theta_{h 1}
\end{array}\right)\left(\begin{array}{l}
d R \\
d \sigma_{1} \\
d t_{1}
\end{array}\right) \\
& \left(\begin{array}{l}
d r \\
d \theta \\
d z
\end{array}\right)=\left(\begin{array}{ccc}
1 & 0 & 0 \\
0 & \sin \theta_{h 2} & -\cos \theta_{h 2} \\
0 & \cos \theta_{h 2} & \sin \theta_{h 2}
\end{array}\right)\left(\begin{array}{l}
d R \\
d \sigma_{2} \\
d_{\tau_{2}}
\end{array}\right)
\end{aligned}
$$

The equations of motion in the intrinsic coordinates can be written as

$$
\left.\begin{array}{l}
\left(\frac{\partial v_{r 1}}{\partial s_{1}}, \frac{\partial v_{\tau 1}}{\partial s_{1}}, \frac{\partial v_{\sigma 1}}{\partial s_{1}}\right)=-\frac{1}{r M_{r}}\left(\frac{\partial P_{r}}{\partial r_{1}}, \frac{\partial P_{r}}{\partial \tau_{1}}, \frac{\partial P_{r}}{\partial s_{1}}\right) \\
\left(\frac{\partial v_{r 2}}{\partial s_{2}}, \frac{\partial v_{\tau 2}}{\partial s_{2}}, \frac{\partial v_{\sigma 2}}{\partial s_{2}}\right)=-\frac{1}{r M_{s}}\left(\frac{\partial P_{s}}{\partial r_{2}}, \frac{\partial P_{s}}{\partial \tau_{2}}, \frac{\partial P_{s}}{\partial s_{2}}\right)
\end{array}\right\}
$$

where $M_{r}$ and $M_{S}$ are the resultant free-stream Mach numbers of the rotor and stator, defined by

$$
\left.\begin{array}{c}
M_{r}^{2}=M^{2}\left(1+\frac{r_{1}^{2}}{R_{\star}^{2}}\right) . \\
M_{s}^{2}=M^{2}\left[1+\left(\frac{r_{1}}{R_{\star}}-\tan \alpha_{2 r}\right)^{2}\right]
\end{array}\right\}
$$

The velocity perturbations $\left(v_{r 1}, v_{g}, v_{\tau}\right)$ and $\left(v_{r} 2\right.$, $v_{02}, v_{\tau 2}$ ) of the rotor and the stator separately can be obtained by integrating Eqs. (35) along a helical streamline by putting $\theta_{h 1}=\left(\theta-z_{1} / R_{\star}\right)$ and integrating over $z_{1}$. We thus obtain the resultant free-stream velocity $\vec{U}_{r}, \vec{U}_{s}$ from the equations

$$
\left\|\begin{array}{l}
U_{R 1} \\
U_{R y^{\prime}} \\
U_{R z^{\prime}}
\end{array}\right\|=\left\|\begin{array}{ccc}
1 & 0 & 0 \\
0 & \sin \alpha_{r} & \cos \alpha_{r} \\
0 & -\cos \alpha_{r} & \sin \alpha_{r}
\end{array}\right\|\left\|\begin{array}{c}
v_{r} \\
v_{\theta}+r_{1} / R_{\star} \\
v_{z}+1
\end{array}\right\|
$$

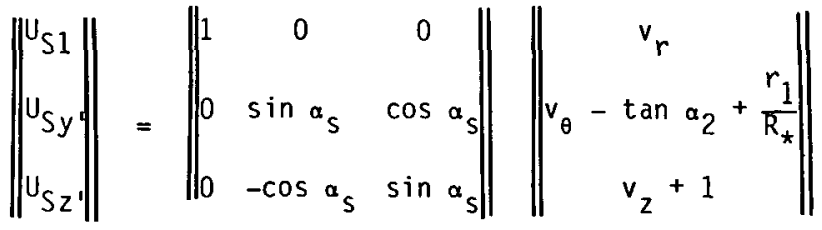

where the total perturbation velocity of the rotorstator combination $\left(v_{r}, v_{\theta}, v_{z}\right)$ is expressed as

$$
\left(\begin{array}{c}
v_{r} \\
v_{\theta} \\
v_{z}
\end{array}\right)=\left(\begin{array}{c}
v_{r r} \\
v_{r \theta} \\
v_{r z}
\end{array}\right)+\left(\begin{array}{c}
v_{s r} \\
v_{s \theta} \\
v_{s z}
\end{array}\right)+\left(\begin{array}{c}
v_{i r} \\
v_{i \theta} \\
v_{i z}
\end{array}\right)
$$

The first two vectors on the right of Eq. (38) are the perturbation velocities of the rotor and stator, each in isolation, and the last vector is the interference velocity due to the simultaneous presence in the compressor stage of the two rows in close proximity. We shall assume that the interference velocity vector is a linear combination of the perturbation velocities due to the rotor and stator, so that Eq. (38) can be written

$$
\left(\begin{array}{c}
v_{r} \\
v_{\theta} \\
v_{z}
\end{array}\right)=(1+\varepsilon)\left(\begin{array}{l}
v_{r r}+v_{s r} \\
v_{r \theta}+v_{s \theta} \\
v_{r z}+v_{s z}
\end{array}\right)
$$

This is the simplest assumption that can be made for the interference effect and permits us to estimate the magnitude of the interaction. By defining the parameters

$\left.\begin{array}{l}\theta_{1}=\theta_{h 1}-\alpha_{r} ; \theta_{3}=\theta_{h 1}-\alpha_{s} ; \varepsilon_{1}=1 /(1+\varepsilon) ; \\ { }_{2}=\theta_{h 2}-\alpha_{r} ; \theta_{4}=\theta_{h 2}-\alpha_{s}\end{array}\right\}$

and combining Eqs. (32) and (40), we can obtain the perturbations $v_{\sigma 1}, v_{\tau 1}, v_{\sigma 2}$, and $v_{\tau 2}$ in the form

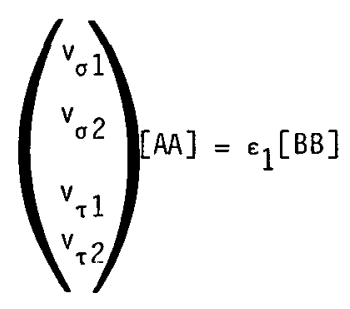


where the matrices $[A A]$ and $[B B]$ are given by

$$
\left.\begin{array}{rl}
A A= & \begin{array}{l}
-\left(\sin \theta_{1}+\tau_{U 1} \cos \theta_{1}\right)-\left(\sin \theta_{2}+\tau_{U 1} \cos \theta_{2}\right)\left(\cos \theta_{1}-\tau_{U 1} \sin \theta_{1}\right)\left(\cos \theta_{2}-\tau_{U 1} \sin \theta_{2}\right) \\
-\left(\sin \theta_{1}+\tau_{L 1} \cos \theta_{1}\right)-\left(\sin \theta_{2}+\tau_{L 1} \cos \theta_{2}\right)\left(\cos \theta_{1}-\tau_{L 1} \sin \theta_{1}\right)\left(\cos \theta_{2}-\tau_{L 1} \sin \theta_{2}\right) \\
-\left(\sin \theta_{3}+\tau_{U 2} \cos \theta_{3}\right)-\left(\sin \theta_{4}+\tau_{U 2} \cos \theta_{4}\right)\left(\cos \theta_{3}-\tau_{U 2} \sin \theta_{3}\right)\left(\cos \theta_{4}-\tau_{U 2} \sin \theta_{4}\right) \\
-\left(\sin \theta_{3}+\tau_{L 2} \cos \theta_{3}\right)-\left(\sin \theta_{4}+\tau_{L 2} \cos \theta_{4}\right)\left(\cos \theta_{3}-\tau_{L 2} \sin \theta_{3}\right)\left(\cos \theta_{4}-\tau_{L 2} \sin \theta_{4}\right)
\end{array}
\end{array}\right\}
$$

The velocity perturbations $v_{\sigma 1}, v_{\sigma 2}, v_{\tau 1}$, and $v_{\tau}$ 2 can be expressed in terms of the coefficients of the Glauert series expansions and written as the system of equations in matrix form

$$
\begin{aligned}
& \left\|\begin{array}{ll}
\mathrm{f}_{1, m} & g_{1, m} \\
\mathrm{f}_{2, m} & g_{2, m} \\
f_{3, m} & g_{3, m} \\
f_{4, m} & g_{4, m}
\end{array}\right\|\left\|\mathscr{A}_{m}^{(k)}\right\|=\varepsilon_{1}\left\|\mathscr{B}_{m}^{(k)}\right\| \mathscr{C}_{\mathscr{C}_{3}} \| \\
& K=0, \pm 1, \pm 2, \ldots, \pm \pm_{\infty}
\end{aligned}
$$

The submatrices $f, g$, and $\mathscr{C}$ in Eq. (44) are functions of the position vector and are not given here because of space limitations but may be obtained from Ramachandra ${ }^{28}$. The coefficients $\mathscr{A}_{\mathrm{m}}^{(k)}$ and $\mathscr{B}_{m}^{(k)}(m=0,1,2, \ldots, \infty)$ are defined in terms of $A_{i}^{(k)}, B_{i}^{(k)}, C_{i}^{(k)}$, and $D_{i}^{(k)}$ as

$\mathscr{A}_{0}^{(\mathrm{k})}=\mathrm{A}_{0}^{(\mathrm{k})}+\mathrm{B}_{0}^{(\mathrm{k})} ; \mathscr{A}_{1}^{(\mathrm{k})}=\mathrm{A}_{1}^{(\mathrm{k})}-2 \mathrm{~B}_{0}^{(\mathrm{k})} ;$

$\mathscr{A}_{\mathrm{m}}^{(\mathrm{k})}=\mathrm{A}_{\mathrm{m}}^{(\mathrm{k})}+\mathrm{B}_{\mathrm{m}}^{(\mathrm{k})} ; \mathscr{B}_{0}^{(\mathrm{k})}=\mathrm{C}_{0}^{(\mathrm{k})}+\mathrm{D}_{0}^{(\mathrm{k})} ;$

$$
\begin{aligned}
\mathscr{B}_{1}^{(k)}=C_{1}^{(k)}-20_{0}^{(k)} ; \mathscr{B}_{m}^{(k)}=C_{m}^{(k)} & +D_{m}^{(k)} \\
m & =2,3, \ldots
\end{aligned}
$$

\section{Rotor-Stator Matching and Final Solution}

With each of the rotor and stator blades is associated a bound vortex of strength $\Gamma_{r}, \Gamma_{s}$ producing the local lift $L=\rho V_{\Gamma}$ per unit span according to the Kutta-Joukowski theorem. The strengths $r_{r}$ and $r_{S}$ of the bound vortex can be obtained from a chordwise integration of $\Delta p_{r}$ and $\Delta \mathrm{p}_{\mathrm{s}}$ as a function of $r_{1}$ and $z_{1}$ as

$$
\begin{gathered}
r_{r_{\infty} \rho_{\alpha} w_{a}}\left(1+\frac{r_{1}^{2}}{R_{\star}^{2}}\right)^{1 / 2}=\int_{\hat{z}_{r 1}}^{\hat{z}_{r 2}} \Delta P_{r} d \hat{z}_{r} \\
r_{s} \rho_{\infty} w_{a}\left[1+\left(\frac{r_{1}}{R_{\star}}-\tan \alpha_{2}\right)^{2}\right]^{1 / 2}=\int_{\hat{z}_{r 1}}^{\hat{z}_{r 2}} \Delta P_{s} d \hat{z}_{s}
\end{gathered}
$$

We assume that the rotor and stator are matched at any operating condition when the net vorticity behind the stator is zero. If Irh and $\Gamma_{\text {sh }}$ are bound vortex strength of the rotor and stator at the blade hub, we express the matching condition by the relation

$$
Z_{r^{r h}}+Z_{s} \Gamma_{s h}=0
$$

By combining Eqs. (24), (29), (45), and (46), we $\mathrm{C}$ an express the matching condition ( $\mathrm{Eq}$. (46)) as

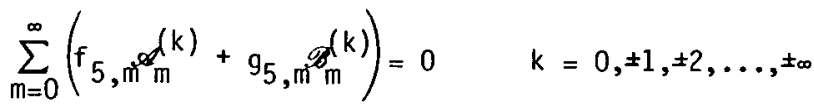

The reader is referred to Ramachandra29 for the expressions of $\mathrm{f}_{5}$ and $g_{5}$ in Eq. (48). A composite equation can be written in matrix form for the unknowns $\mathscr{A}_{\mathrm{m}}, \mathscr{B}_{\mathrm{m}}$, and $\mathscr{E}_{1}$ by combining Eqs. (44) and (48) as

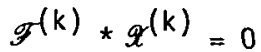


where $\mathscr{T}^{(k)}$ is the vector of unknowns

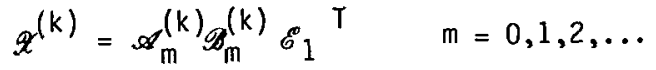

and $\mathscr{F}(k)$ is the matrix

$$
\mathscr{F}^{(k)}=\left\|\begin{array}{lll}
f_{1, m} & g_{1, m}-\mathscr{C}_{1} \\
f_{2, m} & g_{2, m} & -\mathscr{C}_{2} \\
f_{3, m} & g_{3, m}-\mathscr{C}_{3} \\
f_{4, m} & g_{4, m}-\mathscr{C}_{4} \\
f_{5, m} & g_{5, m}-0
\end{array}\right\|_{m=0,1,2, \ldots}
$$

The eigenvectors of the matrix $\mathscr{F}$ in Eq. (49) give the coefficients $\mathscr{A}^{(\mathrm{k})}, \mathscr{B}(\mathrm{k})$, and $\mathscr{E}_{1}$ $\{K=0, \pm 1, \pm 2, \ldots, \pm \infty)$ from which the pressure loading $\Delta \mathrm{p}_{\mathrm{r}}$ and $\Delta \mathrm{p}_{\mathrm{s}}$ on the blades is obtained. Equations (45) are insufficient to determine the coefficients A, B, C, and D of the Glauert series of Eq. (29) in order to separate the thickness and camber contributions.

\section{Combined Pressure Field}

It is now possible to express the pulsating pressure fields $P_{R}$ and $P_{S}$ of the rotor and stator from $\mathrm{Eq}$. (23) inclusive of the interaction effects. The resultant pressure $P$ at any point in the flow is the sum of the pressures $P_{R}$ and PS

$$
\begin{aligned}
P\left(\vec{r}_{1}, t_{1} ; k_{r}, k_{s}, l ; \omega_{r}, \omega_{s}\right) & =P_{R}\left(\vec{r}_{1}, t_{1} ; k_{r}, l ; \omega_{r}\right) \\
& +P_{S}\left(\vec{r}_{1}, t_{1} ; k_{s}, l ; \omega_{s}\right)
\end{aligned}
$$

Assuming that the free-stream values of the streamwise components of the perturbation velocities $v_{\sigma 1, \infty}=v_{\sigma 2, \infty}=0$, we can write Eq. (52) in the form

$$
\begin{aligned}
& \left(4 \pi \beta / Z_{r}\right) P\left(\vec{r}_{1}, t ; k_{r}, k_{s}, l ; \omega_{r}, \omega_{s}\right) \\
& =\sum_{j, l, m=0}^{\infty} \mathscr{R Q R e}{ }^{i \omega_{r} t_{1}-i \mathscr{P}\left(4 B^{3} M Z_{s} / Z_{r}\right) e^{i \omega_{s} t_{1}}}
\end{aligned}
$$

where

$$
\mathscr{U}=\mathscr{U}_{1} \mathscr{U}_{2} ; \mathscr{V}=\mathscr{V}_{1} \mathscr{V}_{2} ; \mathscr{R}=\mathscr{R}_{1} \mathscr{R}_{2} \mathscr{R}_{3} ; \mathscr{S}=\mathscr{S}_{1} \mathscr{S}_{2} \mathscr{S}_{3}
$$

The terms $\left(\mathscr{R}_{1}, \mathscr{S}_{1}\right),\left(\mathscr{R}_{2}, \mathscr{S}_{2}\right)$, and $\left(\mathscr{R}_{3}, \mathscr{S}_{3}\right)$ are, respectively, functions of $r_{1}, \theta$, and $z_{1}$ alone; $\mathscr{U}_{1}, \mathscr{V}_{1}, \mathscr{U}_{2}$, and $\mathscr{V}_{2}$ are constants obtained in terms of the geometry of the stage, the number of blades, and the flow conditions. Again, because of space limitations, the reader is referred to Ramachandra 28 for detailed expressions of these quantities.

The acoustic modes and their characteristics, including their attenuation and resonance properties in ducts, can be obtained from Eq. (53). However, these discussions will be given in separate papers el sewhere.

\section{Discussions and Conclusions}

A lifting surface theory has been formulated for the two rotor-stator blade rows of an axial compressor stage in terms of the acceleration potential. Since the acceleration potential equals the ratio of the perturbation pressure and the mean flow density, the method is equivalent to the determination of the acoustic field of a rotor-stator combination in a duct of inf inite length in terms of the flow Mach number, the geometry, and the oscillation frequency of a distribution of pulsating pressure dipoles through a lifting surface theory. The combined effects of blade thickness and camber have been included as well as that of incidence although it has not been possible to separate them: Curiously, it has been possible to separate their effects when the fluid is incompressible 27 . The perturbation pressures designated in terms of their mode numbers ( $k_{r}, k_{s}$, and $\ell$ ) form a superposition of an inf inite number of acoustic modes consisting of the circumferential wave numbers $k_{r}$ and $k_{s}$ and the radial mode numbers $\ell$. It has also been possible to isolate the rotor-stator interaction effects by matching the blade rows to give zero vorticity downstream of the stator as in an ideal combination. This assumption may appear restrictive in a multistage compressor. However, this can be relaxed and the same method can be used to determine the coefficient vector by using a finite prescribed wake vorticity condition in Eq. (47). From this general solution it is possible to obtain special cases of interest like that of a single or twin tandem actuator disk or single or twin twodimensional cascade rows. This procedure could be extended to the study of counterrotating prope $1-$ lers, of course, by modifying the end conditions in Eq. (20) at the blade tip. This results in a different set of eigenvalues and the corresponding eigenfunctions.

\section{APPENDIX}

In this appendix expressions for the quantities mentioned in the paper are given without their detailed derivations. The functions $I$ and $k$ are, respectively, modified Bessel and Hankel functions of the first kind normalized over the range

$h_{r} \leq r_{1} \leq 1$. The radial eigenfunction $\left(k_{r}\right)$ given in Eq. (A1) appears somewhat different from the form used by McCune ${ }^{33}$ for the rotor. The radial eigenfunctions $\phi\left(k_{r}\right)$ and ${ }_{\psi}\left(k_{s}\right)$ of the rotor and stator are given by

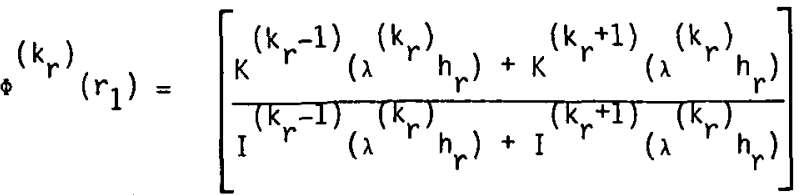

$$
\begin{aligned}
& \times I^{\left(k_{r}\right)}\left(\lambda^{\left(k_{r}\right)} r_{1}\right)+k^{\left(k_{r}\right)}\left(\lambda^{\left(k_{r}\right)_{\left.r_{1}\right)}}\right. \\
& \Psi^{\left(k_{s}\right)}\left(r_{2 s}\right)=\hat{c}^{\left(k_{s}\right)}{ }_{\left(x^{(2 \alpha)}\left(k_{s}\right)\right.}^{(2-1 / 2)^{\left(r_{2 s}\right)}} \\
& \left.+L_{\left(x^{(2 \bar{\alpha})}\left(k_{s}\right)\right.}+\bar{\alpha}-1 / 2\right)^{\left(r_{2 s}\right)}
\end{aligned}
$$


where

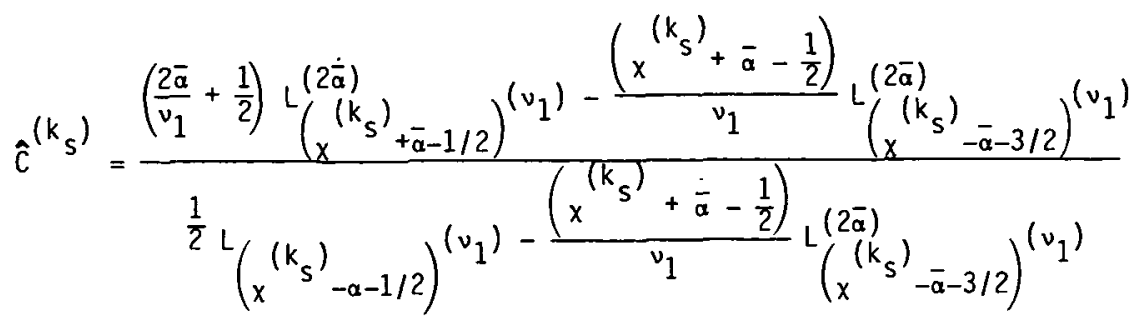

The eigenvalues ${ }_{\lambda}\left(k_{r}\right)$ and $x^{\left(k_{s}\right)}$ are obtained as

$\left[K^{\left(k_{r}-1\right)}\left(\lambda_{1}\right)+K^{\left(k_{r}+1\right)}\left(\lambda_{1}\right)\right]\left[I I_{r}-1\right)\left(\lambda_{\lambda}\left(k_{r}\right)\right)$

$$
\begin{gathered}
\left.{ }_{I}^{\left(k_{r}+1\right)}\left({ }_{\lambda}\left(k_{r}\right)\right)\right]-\left[K^{\left(k_{r}-1\right)}\left({ }_{\lambda}\left(k_{r}\right)\right)+K^{\left(k_{r}+1\right)}\left(\lambda_{\lambda}\left(k_{r}\right)\right)\right] \\
\times\left[I^{\left(k_{r}-1\right)}\left(\lambda_{1}\right)+I^{\left(k_{r}+1\right)}\left(\lambda_{1}\right)\right]=0
\end{gathered}
$$

$\left[\left(\frac{2 \bar{\alpha}}{\nu_{1}}+\frac{1}{2}\right){ }^{(2 \bar{\alpha})}\left(x^{\left(k_{s}\right)}+\bar{\alpha}-1 / 2\right){ }^{\left(\nu_{1}\right)}-\frac{x^{\left(k_{s}\right)}+\bar{\alpha}-\frac{1}{2}}{v^{\left(k_{s}\right)_{R}}}\right.$

$\Phi_{\star}\left(\vec{r}_{1}, \vec{p}_{r}, t_{1} ; k_{r}, l\right)=\frac{\Phi_{\ell}^{\left(k_{r}\right)}\left(\rho_{r}\right)_{\ell}^{\left(k_{r}\right)}\left(r_{1}\right)}{\Lambda_{\ell}\left(k_{r}\right)}$

$x\left[\frac{i k_{r}}{\rho_{r}}+\tan a_{r}\left(\frac{\Lambda_{\ell}{ }^{\left(k_{r}\right)}}{\beta} \operatorname{sgn} \hat{Z}_{r}-i M \hat{M} \hat{Z}_{r}\right)\right]$

$\times \exp \left[i M \hat{M} \hat{Z}_{r}-\frac{\Lambda_{l}^{\left(k_{r}\right)}}{\beta}\left|\hat{Z}_{r}\right|\right.$

$x \exp i\left[k_{r}\left(\theta-\bar{\varphi}_{r}-\varphi_{r}\right)+\omega_{r} t_{1}\right]$

$\left.\times{ }^{(2 \bar{\alpha})}\left(x^{\left(k_{s}\right)}-\bar{\alpha}-3 / 2\right)^{\left(v_{1}\right)}\right] \times\left[\frac{1}{2} L^{(2 \bar{\alpha})}\left(x^{\left(k_{s}\right)}-\bar{\alpha}-1 / 2\right)\right.$

$x\left(v^{\left(k_{s}\right)} R_{s r}\right)-\frac{x^{\left(k_{s}\right)}+\bar{\alpha}-\frac{1}{2}}{v^{\left(k_{s}\right)}{ }_{R}}\left({ }^{(2 \bar{\alpha})}\left(x_{s r}^{\left(k_{s}\right)}-\bar{\alpha}-3 / 2\right)^{\left(v^{\left(k_{s}\right)} R_{s r}\right)}\right]$

$y_{\star}\left(\vec{r}_{1}, \vec{p}_{s}, t_{1} ; k_{s}, l\right)=\exp \left[-\frac{\left(r_{2 s}+\rho_{2 s}\right)}{2}\right]\left(r_{2 s} \rho_{2 s}\right)^{\bar{\alpha}+1 / 2}$

$\times \psi_{\ell}^{\left(k_{s}\right)}\left(\rho_{2 s}\right) \psi_{\ell}^{\left(k_{s}\right)}\left(r_{2 s}\right)$

$+\left[\left(\frac{2 \bar{\alpha}}{\left(v_{s}\right)_{R_{s r}}}+\frac{1}{2}\right) L\left(x^{(2 \bar{\alpha})}\left(k_{s}\right)_{+\bar{\alpha}-1 / 2}\right)^{\left(v_{s}^{\left(k_{s}\right)_{R_{s r}}}\right)}\right.$

$\times\left[\left[-\frac{i k_{S}}{\rho_{S}}\left(C F \cos M \Delta_{1} \hat{Z}_{s}+S F \sin M \Delta_{1} \hat{Z}_{s}\right)+M \tan \alpha_{S}\right.\right.$

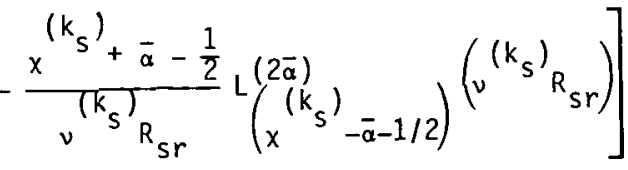

$\times\left\{\Delta_{1}\left(S F \cos M \Delta_{1} \hat{z}_{s}-C F \sin M \Delta_{1} \hat{z}_{s}\right)\right.$

$+\left[i M_{\star}\left(i \mathscr{R e \Delta _ { 2 }}-\left|\int m \Delta_{2}\right| \operatorname{sgn} \hat{z}_{s}\right]\left(C F \cos M_{\Delta_{1}} \hat{z}_{s}\right.\right.$

$\left.\left.\left.+S F \sin M \Delta_{1} \hat{Z}_{s}\right)\right\}\right] \exp \left[i M M_{\star} \hat{Z}_{s}+M\left(i \mathscr{R e} \Delta_{2}\right.\right.$

$\left.-\mid\left(m \Delta_{2} \mid\right) \hat{z}_{s}\right] \exp i\left[k_{s}\left(\theta-\bar{\varphi}_{s}-\varphi_{s}\right)\right] \times \exp \left(i_{s} t_{1}\right)$

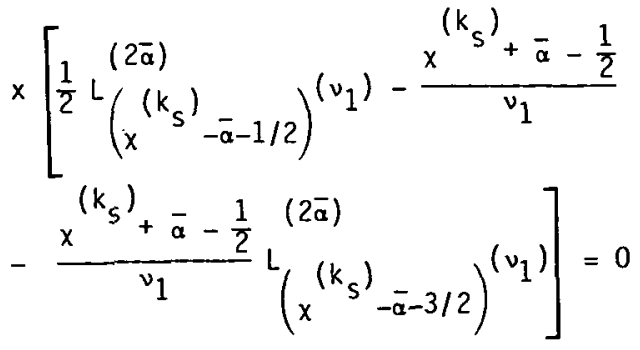

where CF and SF are functions defined in Ramachandra 28 and will not be given here because of their lengthy nature.

(A5) 


\section{References}

1. Kemp, N. H. and Sears, W. R., "Aerodynamic Interference Between Moving Blade Rows," Journal of the Aeronautical Sciences, vol. 20, Sept. 1953, pp. 585-597.

2. Kemp, N. H. and Sears, W. R.," The Unsteady Forces Due to Viscous Wakes in Turbomachines," Journal of the Aeronautical Sciences, vol. 22, JuTy 1955, pp. 478-483.

3. Lane, F. and Friedman, M., "Theoretical Investigation of Subsonic 0scillatory Blade-Row Aerodynamics," NACA TN 4136, 1958.

4. Carta, F. 0., "Unsteady Aerodynamic Theory of a Staggered Cascade of Oscillating Airfoils in Compressible Flow, "United Aircraft Corp. Res. Rep. UAC R-0582-19, 1957.

5. Carta, F. 0. and Fanti, R., "Aerodynamic Interaction Effects of Cascaded Airfoils Oscillating in Two Dimensional Compressible Flow," United Aircraft Corp. Res. Rep. UAC R-0582-17, 1957 .

6. Hetherington, R., "Compressor Noise Generated by Fluctuating Lift Resulting from RotorStator Interaction," AIAA Journal, vol. 1, Feb. 1963, pp. 473-474.

7. Parker, R., "Resonance Effects in Wake Shedding from Parallel Plates: Some Experimental 0bservations," Journal of Sound and Vibration, vol. 4, July 1966, pp. 62-72.

8. Parker, R., "Resonance Effects in Wake Shedding from Parallel Plates: Calculation of Resonant Frequencies, "Journal of Sound and Vibration, vol. 5, March 1967, pp. $330-343$.

9. Parker, R. and Pryce, D. C., "Wave Excited Resonances in an Annular Cascade: An Experimental Investigation," Journal of Sound and Vibration, vol. 37, Nov. 1974, pp. 247-261.

10. Tyler, J. M. and Sofrin, T. G., "Axial Flow Compressor Noise Studies," SAE Preprint 345D, Society of Automotive Engineers, 1961.

11. Tyler, J. M. and Sofrin, T. G., "Axial Flow Compressor Noise Studies," SAE Transactions, vol. 70,1962 , p. 309 .

12. Smith, S. N., "Discrete Frequency Sound Generation in Axial Flow Turbomachines, "ARC R\&M 3709, 1973.

13. Whitehead, D. S., "Vibration and Sound Generation in a Cascade of Flat Plates in Subsonic Flow," ARC R\&M 3685, 1970.

14. Morfey, C. L., "Sound Generation in Subsonic Turbomachinery," Transactions of the ASME Journal of Basic Engineering, Ser. D, voT. 92, Sept. 1970., pp. 450-458.
15. Morfey, C. L., "A Review of Sound Generating Mechanisms in Aircraft Engine Fans and Compressors," Aerodynamic Noise, Proceedings of the Symposium, edited by H. S. Ribner, Univ. of Toronto Press, Toronto, 1969, pp. 299-329.

16. Morfey, C. L., "Rotating Pressure Patterns in Ducts: Their Generation and. Transmission," Journal of Sound and Vibration, vol. 1, Jan. 1964, pp. 60-87.

17. Mani, R., "Compressibility Effects in the Kemp and Sears Problem," Fluid Mechanics, Acoustics and Design of Turbomachinery, Part II, edited by B. Lakshminarayana, W. R. Britsch, and W. S. Gearhart, NASA SP-304, 1974, pp. 513-536.

18. Mani, R., "Discrete Frequency Noise Generation from an Axial Flow Fan Blade Row," Transactions of the ASME, Journal of Basic Engineering, Ser. D, vol. 93, Mar. 1970, p. 37 .

19. Mani, R. and Horvay, G., "Sound Transmission Through Blade Rows", Journal of Sound and Vibration, vol. 12, May 1970, pp. 59-83.

20. Kaji, S. and Okazaki, T., "Propagation of Sound Through a Blade Row. Part I. Analys is Based on the Semi-Actuator Disk Theory," Journal of Sound and Vibration, vol. 11, Mar. 1970, pp. 339-353.

21. Kaji, S. and Okazaki, T, "Propagation of Sound Waves Through a Blade Row. Part II . Analys is Based on the Acceleration Potential Method," Journal of Sound and Vibration, vol. 11, Mar. 1970, pp. 355-375.

22. Kaji, S. and Okazaki, T., "Generation of Sound by Rotor-Stator Interaction," Journal of Sound and Vibration, vol. 13, Nov. 1970, pp. 281-307.

23. Slutsky, S., "Discrete Noise Generation and Propagation by a Fan Engine," Aerodynamic Noise, Proceedings of the Symposium, edited by H. S. Ribner, Univ. of Toronto Press, Toronto, 1969, pp. 331-349.

24. Namba, M., "Three-Dimensional Analys is of Blade Force and Sound Generation for an Annular Cascade in Distorted Flows," Journal of Sound and Vibration, vol. 50, Feb. 1977, pp. 479-508.

25. Namba, M., "Lifting Surface Theory for a Rotating Subsonic or Transonic Blade Row, " ARC R\&M 3740, 1974.

26. Marjano, S., "Ground Effect of a Plane Uniform Sound Source Distribution," Journal of Sound and Vibration, vol. 11, Jan. 1970, pp. 145-148.

27. Ramachandra, S. M., "Lifting Theory for a Turbomachine Stage." NASA TM to be published. 
28. Ramachandra, S. M., "The Combined Pressure Field of an Axial Compressor Stage." NASA TM to be published.

29. Flugge, S., ed., "Handbuch Der Physik, Vol. 1, Mathemat ische Methoden 1, "(Encyclopedia of Physics, vol. 1, Mathematical Methods 1), Springer-Verlag, Berlin, 1956.

30. Magnus, W., Oberhettinger, F., and Soni, R. P., "Formulas and Theorems for the Special Functions of Mathematical Physics," Springer-Verlag, New York, 1966.
31. Szego, Gabor, "Orthogonal Polynomials," American Mathematical Society, New York, 1959.

32. Kotorynski, W. P., "On an Irregular Sturm-Liouville Problem in Boundary Layer Theory, "SIAM Journal of Applied Mathematics, vol. 16, Nov. 1968, pp. 1132-1140. 


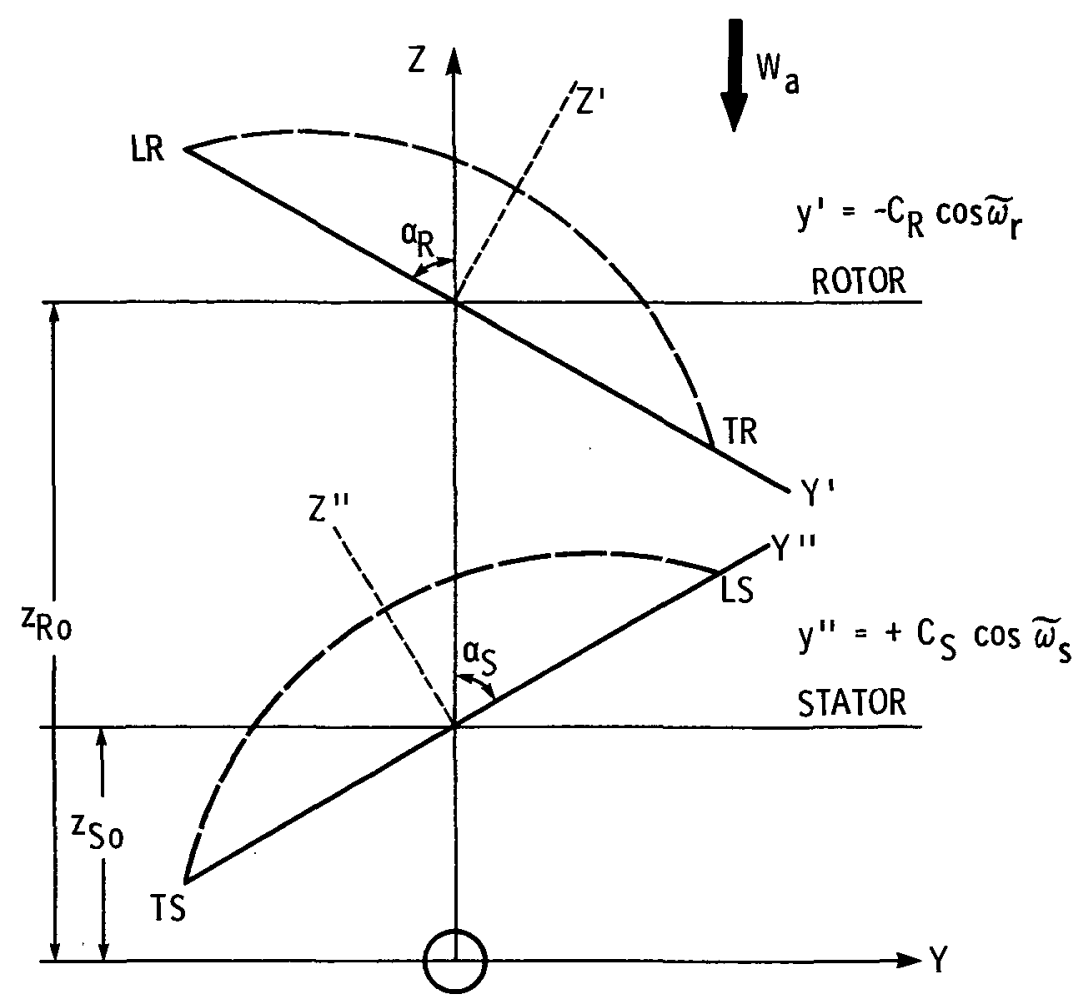

Fig. 1 Local coordinate system for blades.

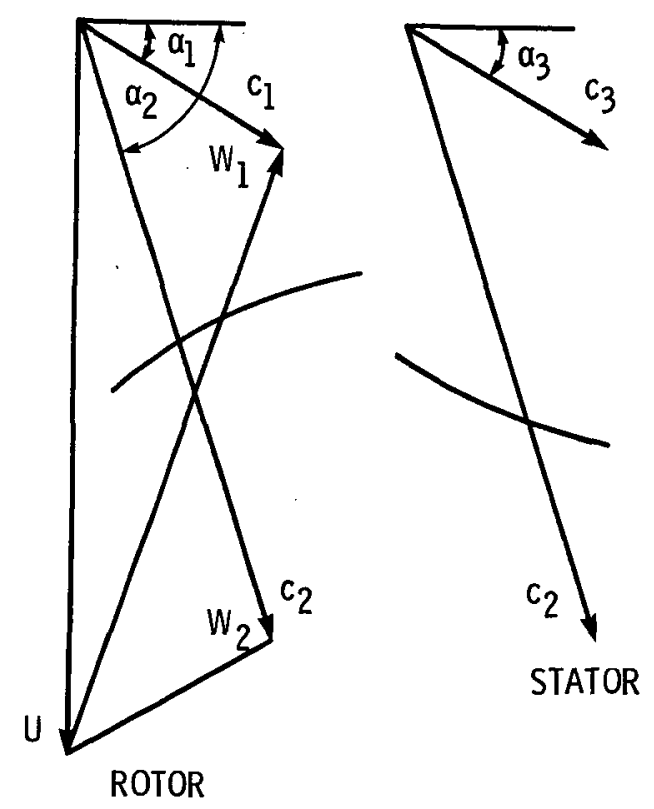

Fig. 2 Velocity diagram of stage. 


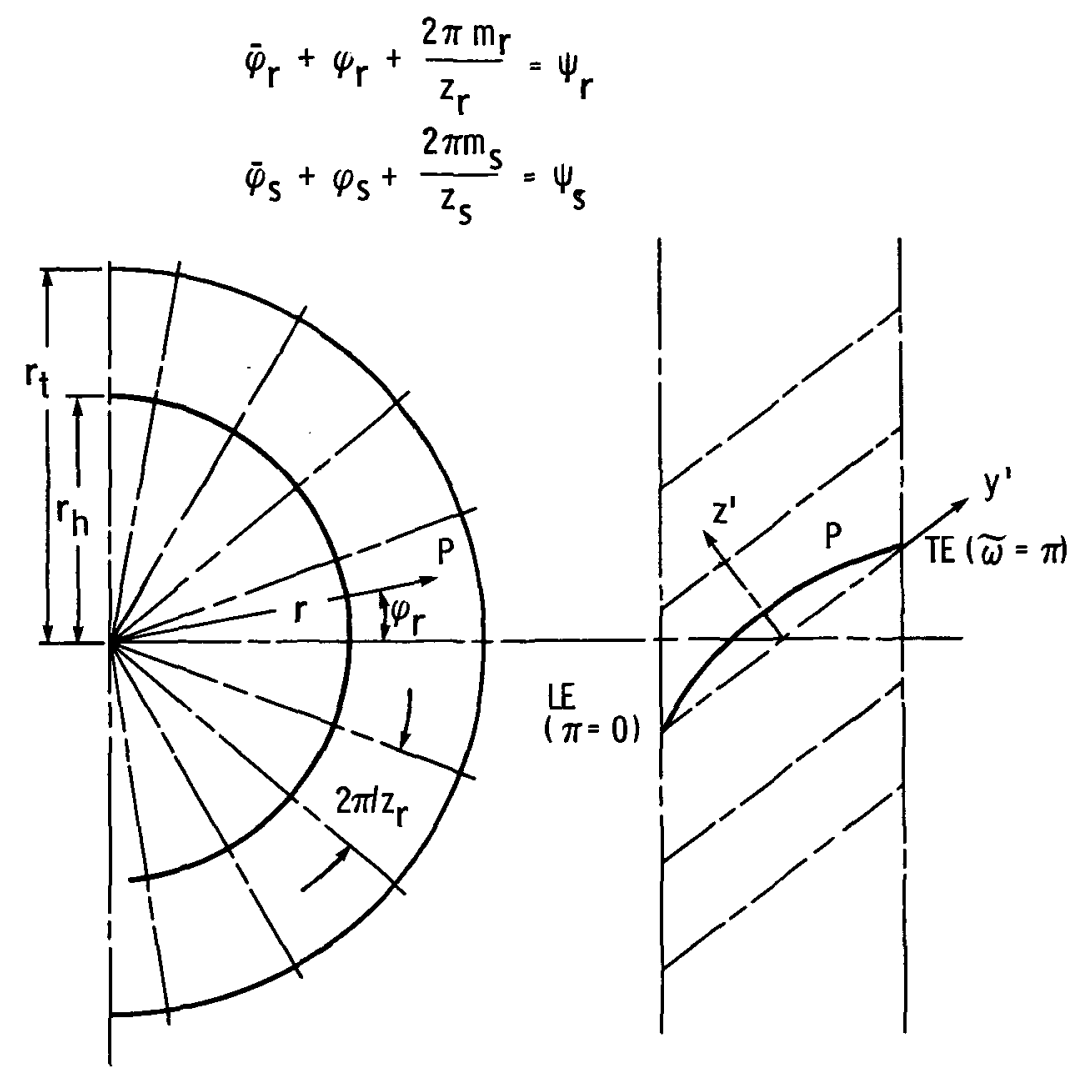

Fig. 3 Schematic diagram of casade.

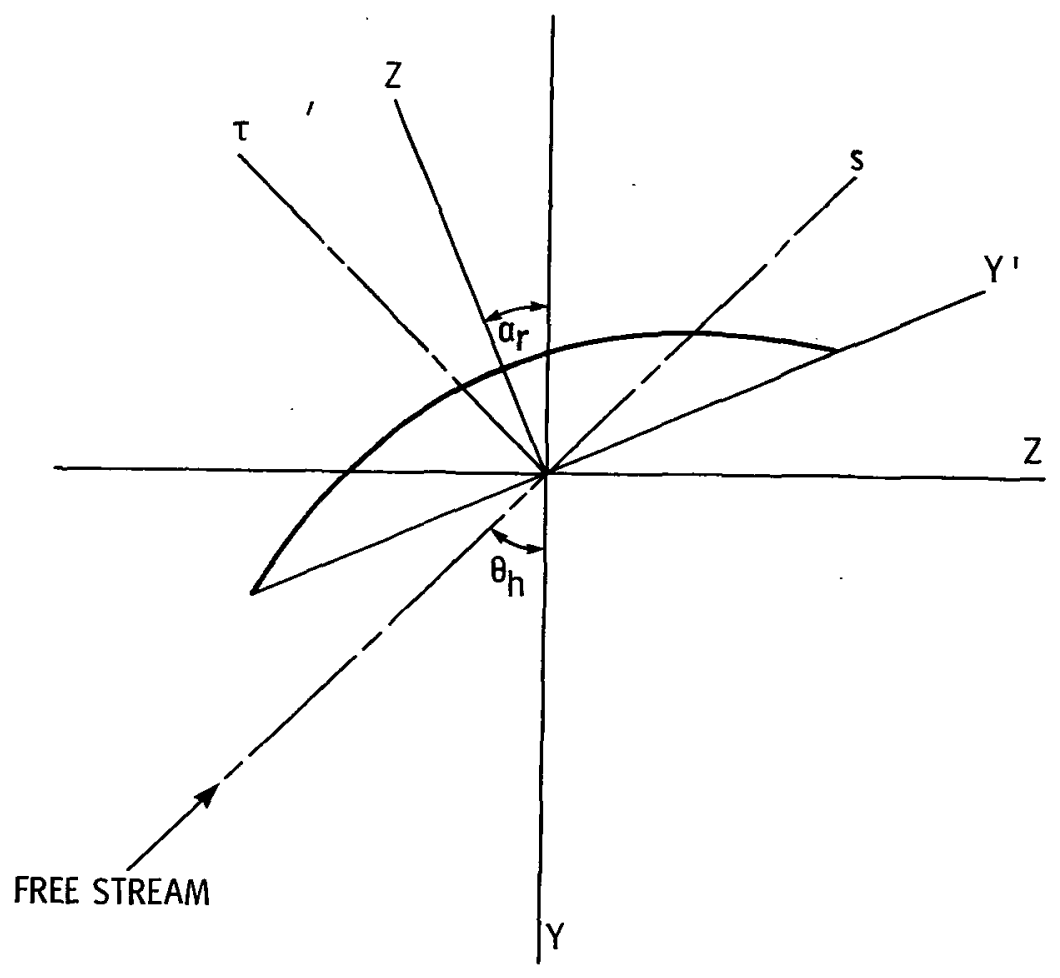

Fig. 4 Helical coordinate system for blade. 


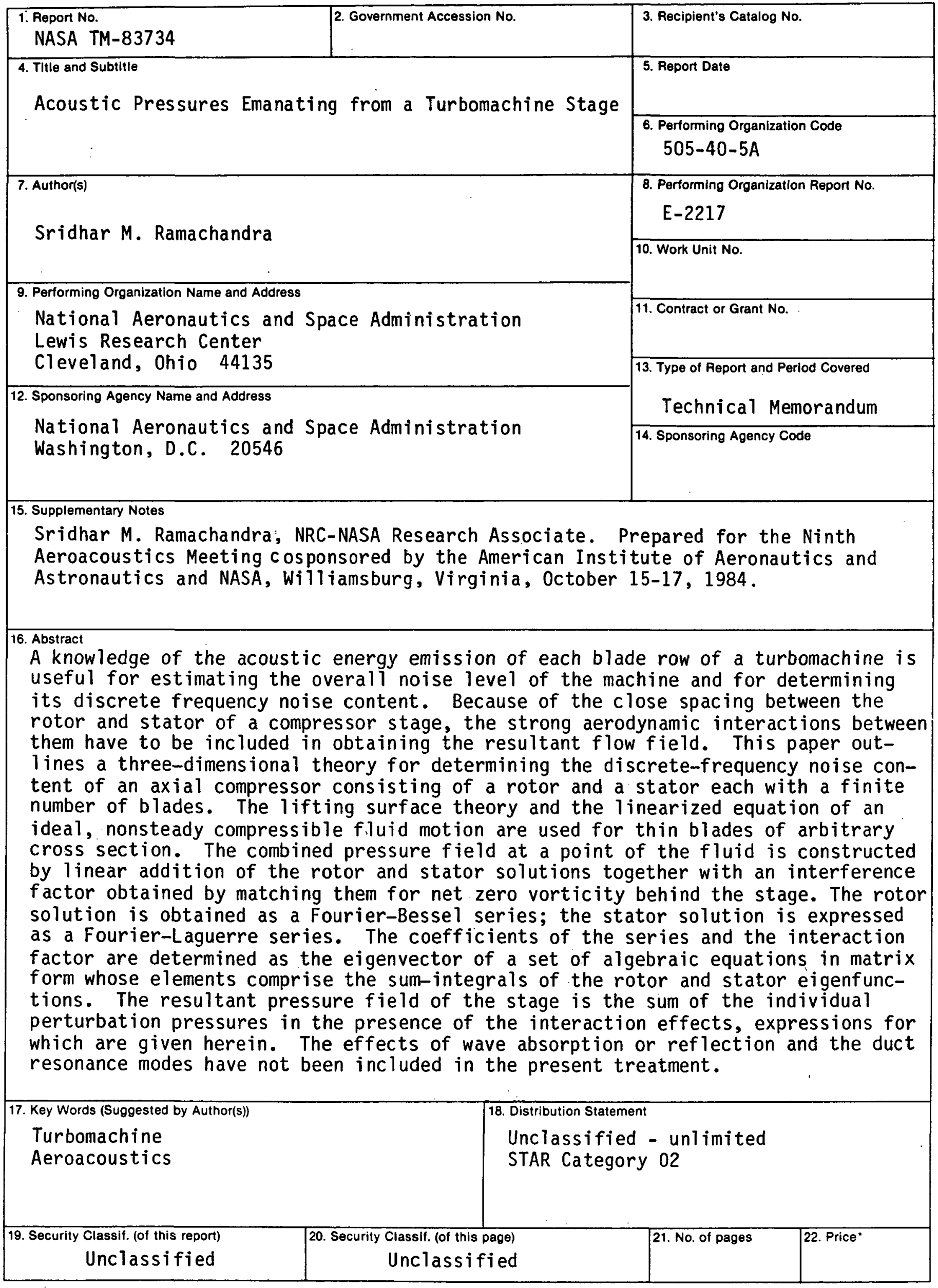

"For sale by the National Technical Information Service, Springfield, Virginia 22161 
National Aeronautics and Space Administration

Washington, D.C.

20546

Oflicial Business

Penally for Private Use, $\mathbf{5 3 0 0}$
SPECIAL FOUATH CLASS MAIL BOOK

Portege and Fees Paid National Aeronautics and Space Administration NASA 451 